\title{
Free surface intake vortices: Theoretical model and measurements
}

FRANK SUERICH-GULICK, Ph. D. Graduate, Department of Civil Engineering and Applied Mechanics, McGill University, Montréal, Canada

SUSAN. J. GASKIN (IAHR Member), Associate Professor, Department of Civil Engineering and Applied Mechanics, McGill University, MacDonald Engineering Bldg, Room 492, Montréal, QC, Canada H3A 2 K6

Email: susan.gaskin@mcgill.ca (author for correspondance)

MARC VILLENEUVE (IAHR Member), President, Lasalle Consulting Group, LaSalle, Canada

ÉTIENNE PARKINSON, Head of Research \& Development Vevey, Andritz Hydro, Vevey, Switzerland

Running Head: Free surface intake vortices: Model and measurements

\begin{abstract}
Axially stretched free surface vortices occur at low-head hydropower intakes under specific flow and geometric conditions. When they are sufficiently strong, they can harm performance or cause premature failure of mechanical components such as turbine blades or guide vanes. Laboratory-scale experimental models are currently used to assess the risk of vortex formation during the design phase, but uncertainty remains as to how vortex characteristics translate from the laboratory scale to the much larger scale of an actual hydropower plant. This paper proposes a semi-empirical model that roughly predicts how the approach flow and intake geometry determine the key vortex characteristics (the core radius, bulk circulation and the depth of the free surface depression). The model is developed using detailed velocity measurements of the approach flow and the flow inside the vortex in a laboratory-scale physical model, using analytical models and insights drawn from previous work.
\end{abstract}

Keywords: axial vortex stretching; hydraulic model; hydraulics of renewable energy systems; hydropower intake; particle tracking velocimetry (PTV); turbulence in rotating flow; vortex dynamics. 
Free surface intake vortices: Theoretical model and measurements

\section{Introduction}

Free surface vortices are a common problem directly upstream of low-head hydropower plants and pumping stations. The structure that guides the flow transition from relatively slow-moving open channel (free surface) flow approaching the plant or station to faster pressure flow entering the submerged pipe or penstock leading to the turbines or pumps is referred to as the intake; the vortices that form at the intake are referred to as intake vortices.

The phenomenon of intake vortices was studied extensively in the 70s and 80s by researchers seeking to predict vortex characteristics and risk of air entrainment at a given flow rate and submergence. Early efforts relied on reduced-scale laboratory experiments (Daggett and Keulegan 1974; Jain et al. 1978; Pennino and Hecker 1979; Anwar 1983) as well as some surveys of vortex activity in full-scale 'prototype' hydropower and pumping intakes (Gordon 1970; Pennino and Hecker 1979; Gulliver et al. 1986). The resulting correlations are still used today by design engineers during the early stages of the design process to estimate the minimum acceptable submergence: this is the minimum distance that the opening of the intake pipe or penstock may be to the free surface for a given intake velocity before problematic vortices occur (Tastan and Yildirim 2010). The predictive accuracy of these correlations is limited by the fact that vortex characteristics such as the characteristic radius, bulk circulation and the tip depth (the total depth of the free surface depression produced by the vortex) are very sensitive to the geometry of the intake structure and to the associated velocity distribution. Physical testing using laboratory-scale models therefore remains a key component of the assessment and optimization process for most large projects. However, a degree of uncertainty remains when interpreting the results of these tests, because the various forces that control vortex characteristics scale differently. Scale effects should thus be carefully considered in physical model tests.

Many attempts have been made to assess and predict scale effects. Daggett and Keulegan (1974), Jain et al. (1978), Anwar (1983), and Tastan and Yildirim (2010) approach the question empirically, identifying the minimum size of a laboratory model (or recommended cut-off values of associated non-dimensional parameters) required for scale effects to be negligible. These recommended cut-off values vary from author to author (Tastan and Yildirim 2010) and may be difficult to meet for large-scale projects such as hydropower intakes within the economic and spatial constraints.

Several analytical models have been developed to describe processes such as vorticity generation, diffusion and axial vortex stretching that govern intake vortex characteristics. Vorticity is a vector quantity $\boldsymbol{\omega}$ that locally quantifies the rate of rotation of a fluid particle about its centre of mass. It is defined mathematically as the curl of the velocity vector: $\boldsymbol{\omega}=\boldsymbol{\nabla} \times \boldsymbol{V}$ (Helmholtz 1867).

The vortex flow is described using a local cylindrical coordinate system $(r, \theta, z)$ with $z$ pointing down from the free surface along the central axis of the vortex, and with corresponding radial, azimuthal and axial velocities $V_{r}, V_{\theta}$ and $V_{z}$. If the axial velocity $V_{z}$ of a vortex increases along its axis, the vortex is said to be axially stretched, and its streamlines converge towards the axis $\left(V_{r}<0\right)$. If viscous losses are negligible, then the vortex's angular momentum is conserved and vorticity in the vortex increases in proportion to the axial gradient (Helmholtz 1867). In most flows, viscosity tends to smear vorticity radially outwards, leading to a Gaussian-like profile (Rott 1958).

Burgers (1948) and Rott (1958) independently developed a vortex model in which an equilibrium 
of axial stretching and viscous diffusion produces a stable vortex with a constant vorticity profile along its axis. They assume that the radial profiles of $V_{\theta}$ and $V_{r}$ are constant along $z$ and that the axial velocity $V_{z}$ is independent of $r$ and increases linearly with $z: V_{z}(z)=a z, V_{r}(r)=-a r / 2$, where the gradient $a$ is a constant with units of $\mathrm{s}^{-1}$ and the profile of $V_{r}$ is set to satisfy continuity. Solving the axisymmetric Navier-Stokes equations with these assumptions produces

$$
V_{\theta}(r)=\frac{\Gamma_{\infty}}{2 \pi r}\left[1-\exp \left\{-\left(r / r_{o}\right)^{2}\right\}\right]
$$

where $\Gamma_{\infty}$ is the bulk circulation, assumed to reach a constant value far from the vortex centre. What will be hereafter referred to as the characteristic radius $r_{o}$ is determined by the ratio of viscosity to axial gradient:

$$
r_{o}=2(v / a)^{1 / 2}, \quad a=\partial V_{z} / \partial z
$$

where $v$ is the kinematic viscosity of the fluid.

Einstein and $\mathrm{Li}$ (1951) applied a modified version of this model to intake vortices (later further adjusted by Bøhling et al. (2010)), defining an inner region in which a Burgers vortex is concentrated, surrounded by an external zone with no axial velocity. Odgaard (1986) used Burgers's (1948) model directly to predict critical submergence for an air core vortex in a cylindrical tank with imposed flow rotation: this work highlighted the link between submergence and the key process of axial stretching (Quick 1970; Carriveau et al. 2009; Petitjeans 2003). Odgaard (1986) assumes that the axial velocity in the central portion of the tank follows a linear profile from zero at the free surface to $U_{i}$ at the bottom of the tank, so that $a=U_{i} / H$, where $H$ is the water depth and $U_{i}$ is the mean outflow velocity. Odgaard's and subsequent results (Hite and Mih 1994; Ito et al. 2010) support the use of Burgers's model for modelling intake vortices, even if it does not capture their full complexity. Other variants of Burgers's model have been proposed by Lundgren (1985),Hite and Mih (1994),Miles (1998), Rossi et al. (2004), Andersen et al. (2006), Stepanyants and Yeoh (2008), Ito et al. (2010), and Wang et al. (2011), among others.

There remains a shortage of detailed measurements of vortices and the flow surrounding them for geometries more closely resembling hydropower intakes, such as Hite and Mih (1994) and Nakayama and Hisasue (2010). These are required to help adapt models of free surface vortices to specific intakes and to better understand how geometry and intake approach flow influence vortex characteristics (Quick 1970; Yildirim et al. 2000; Ansar and Nakato 2001). Computational fluid dynamics models have potential to help predict the flow field approaching intakes, but they remain expensive and insufficiently validated for modelling the vortices themselves with adequate accuracy within the time and economic constraints of an hydraulic industrial context. This paper builds on Odgaard's (1986) model by first testing its hypotheses using experimental data collected in a laboratory model and then adapting it to incorporate the influence of the intake geometry. Detailed velocity measurements are taken to establish the relationship between the geometry, the flow approaching the intake pipe and the characteristics of the vortices that form. The measurements are then used to adapt Burgers's vortex model to the specific intake geometry studied in the laboratory.

\section{Experimental setup}

A laboratory-scale experimental model is constructed that reproduces the flow configuration of a simplified low-head hydropower intake with approximately uniform approach flow conditions. As shown in Fig. 1, the 
channel has a square 1 by $1 \mathrm{~m}$ cross-section with a $3.9 \mathrm{~m}$ long flat bed and a circular 'intake' pipe of inner diameter $d=11.5 \mathrm{~cm}$ mounted flush into the downstream wall of the channel, with its axis located $0.14 \mathrm{~m}$ above the channel bed. This is a simplification compared to most hydropower intakes, where the intake opening is usually more rectangular in shape.

(a)
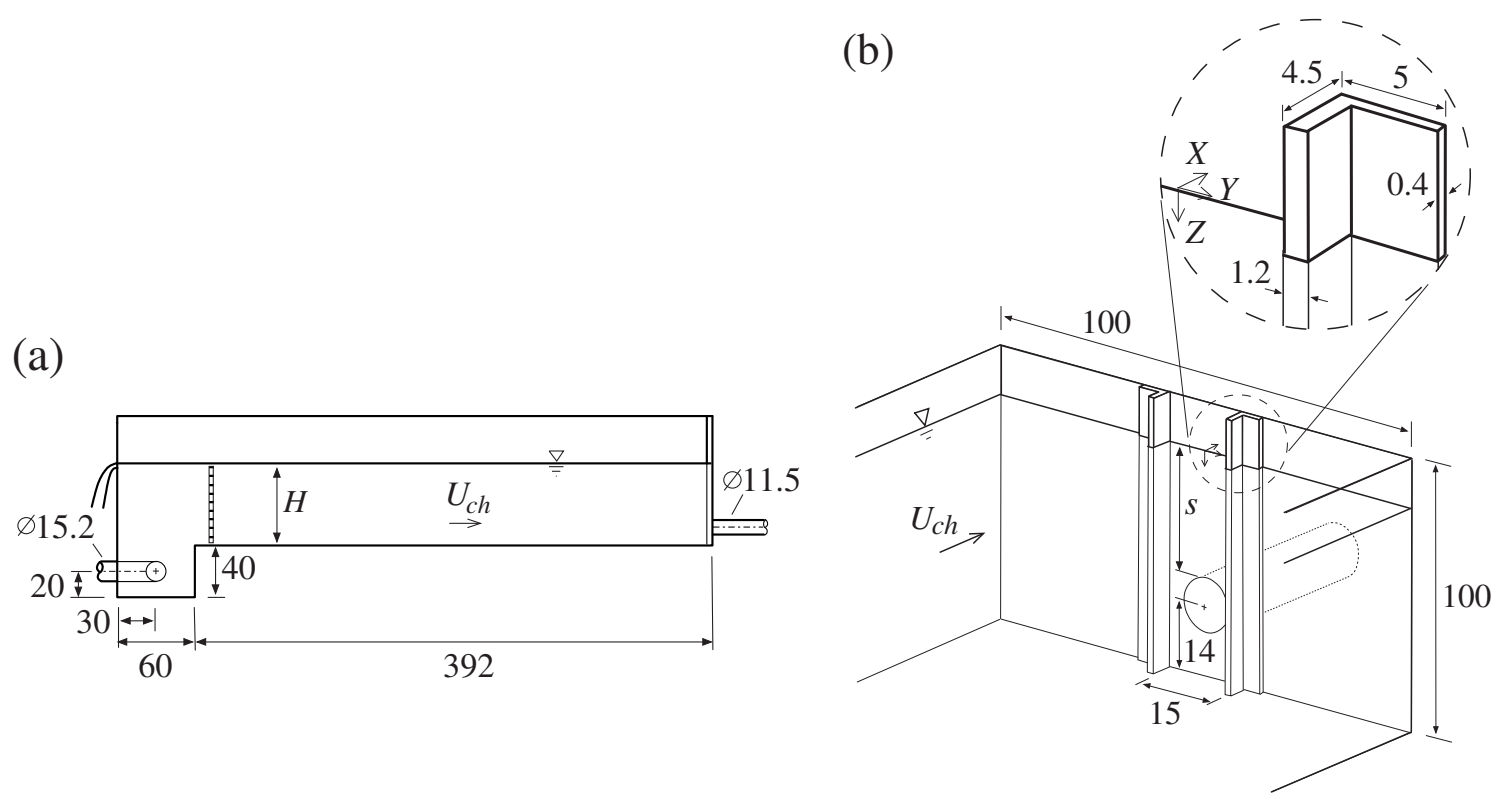

Figure 1 (a) Vertical section and (b) isometric views of the laboratory model (dimensions in $\mathrm{cm}$ ). Adapted from Suerich-Gulick et al. (2014a)

Two narrow piers are mounted perpendicular to the downstream wall on each side of the intake pipe. Each pier generates a relatively stable vortex pair in its wake (Hite and Mih 1994) with one vortex starting at the free surface and one starting from the floor of the tank; both vortex tails are entrained into the intake pipe as shown in Fig. 2a. Similar piers are found at hydropower intakes where they hold the trash-racks across the penstock opening (Pennino and Hecker 1979; Gulliver et al. 1986; Jiming et al. 2000; Montilla et al. 2004). The piers in the experiment protrude further into the flow and are less streamlined to produce more stable vortices and a clearly defined separation zone, thus easing measurements and analysis. Figure $2 \mathrm{~b}$ shows a top view schematic of the free surface streamlines in the experiment, with the piers and back wall shown in grey. Only the free surface vortices are examined in this paper since their presence and effects are of greater concern to plant operators. The two piers are spaced $k=15 \mathrm{~cm}$ apart, symmetrically about the pipe axis; their cross-section is rectangular, $1.2 \mathrm{~cm}$ thick and $4.5 \mathrm{~cm}$ long and they span the full channel depth (Fig. 1b).

The water level in the channel is controlled by the back-pressure at the exit of the intake pipe and it is stabilized with a square-crested weir mounted into the upstream wall of the channel. The water is injected into the tank through a T-shaped diffuser submerged in a $60 \mathrm{~cm}$ long, $40 \mathrm{~cm}$ deep reservoir directly upstream of the channel. Large eddies produced as the flow exits the diffuser are broken down with a 1.9 $\mathrm{cm}(3 / 4 ")$ thick sheet of aluminum honeycomb placed just downstream of the step from the reservoir to the channel. A global coordinate system $(X, Y, Z)$ is defined with its origin at the free surface, half-way across the downstream wall of the channel. $Z$ points down towards the bed, $X$ points downstream and $Y$ points towards the right when looking downstream. 
(a)

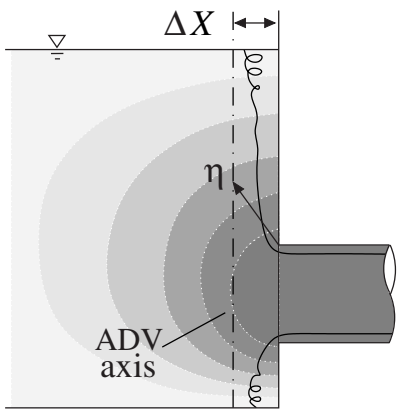

(b)

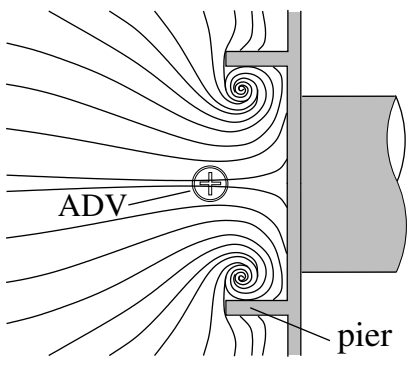

Figure 2 (a) Schematic side view of the intake approach flow on the vertical plane along the channel centerline, with shading indicating velocity magnitude. Adapted from Suerich-Gulick et al. (2014a). (b) Top view sketch of streamlines separating off the pier tips.

Eight combinations of submergence and intake velocity are selected to produce a wide range of vortex intensities (see Table 1). The intake velocity ranges from $U_{i}=0.58$ to $1.80 \mathrm{~ms}^{-1}$ and the relative submergence ranges from $s / d=1.5$ to 3.3 , where $U_{i}=4 Q /\left(\pi d^{2}\right)$ is the mean velocity in the intake pipe and $s$ is the vertical distance from the free surface to the top of the pipe opening (as shown in Fig. 1b). The mean channel velocity $U_{c h}=Q /(b H)$ ranges from 16 to $40 \mathrm{mms}^{-1}$, where $H$ is the nominal water depth in the channel and $b=1 \mathrm{~m}$ is the channel width. The deepest submergence studied $(s / d=3.3)$ is the approximate transition point where the vortices become much weaker and more sporadic. $R_{s}=U_{i} s / v$, $\mathrm{F}_{\mathrm{S}}=U_{i} /(g s)^{1 / 2}$ and $\mathrm{W}=\rho U_{i}^{2} s / \sigma$ are the submergence Reynolds, Froude and Weber numbers respectively, and $\mathrm{R}_{\mathrm{ch}}=U_{c h} H / v$ is the channel Reynolds number. The experiment is operated at temperatures ranging from 12 to $15^{\circ} \mathrm{C}$.

\subsection{Measurements}

For each operating condition, three different measurement methods are used to document the flow conditions upstream from the intake and directly in front of it, as well as the velocity field inside the vortex and the free surface depression it produces. These data allow us to clarify how the intake geometry controls the structure of the flow directly in front of it and how it thereby controls the vortex characteristics. The measurements are then used to guide the choice of analytical relations and coefficients used to relate the vortex characteristics to the intake configuration. The data are collected using an acoustic Doppler velocimeter (ADV) and highspeed films that simultaneously record the free surface profile and particle trajectories for particle tracking velocimetry (PTV).

\subsection{ADV measurements}

A Sontek Micro-ADV is used to record the larger-scale structure of the flow approaching the intake and to quantify how the velocity fields outside the vortex control the circulation and the characteristic radius of the vortex through axial stretching. Mean velocities $U_{X}, U_{Y}$ and $U_{Z}$ are measured at $30 \mathrm{~Hz}$ and averaged over two minutes. Coarse measurement grids are taken $2 \mathrm{~m}$ and $0.2 \mathrm{~m}$ upstream of the intake and a vertical line of more closely-spaced measurements is taken $5.5 \mathrm{~cm}$ upstream from the intake pipe on the channel centreline 
Table 1 Operating conditions for the measurements

\begin{tabular}{ccccccccc}
\hline Case & $s / d$ & $U_{i}$ & $U_{c h}$ & $\begin{array}{c}\mathrm{R}_{\mathrm{s}} \\
\times 10^{-5}\end{array}$ & $\begin{array}{c}\mathrm{R}_{\mathrm{ch}} \\
\times 10^{-4}\end{array}$ & $\begin{array}{c}\mathrm{F}_{\mathrm{s}} \\
\left(\mathrm{ms}^{-1}\right)\end{array}$ & $\begin{array}{c}\mathrm{W} \\
\times 10^{-3}\end{array}$ & $\begin{array}{c}\text { vortex } \\
\text { type* }\end{array}$ \\
\hline 1 & 1.5 & 0.58 & 16 & 0.8 & 0.5 & 0.4 & 0.8 & 4 \\
2 & 1.5 & 0.86 & 24 & 1.2 & 0.7 & 0.7 & 1.8 & 4 \\
3 & 1.5 & 1.40 & 39 & 2.0 & 1.2 & 1.1 & 4.8 & 5 \\
4 & 2.4 & 0.86 & 19 & 1.9 & 0.7 & 0.5 & 2.8 & 4 \\
5 & 2.4 & 1.80 & 40 & 4.1 & 1.5 & 1.1 & 12.4 & 4 \\
6 & 3.3 & 0.86 & 16 & 2.7 & 0.7 & 0.4 & 3.9 & $1-3$ \\
7 & 3.3 & 1.40 & 25 & 4.3 & 1.2 & 0.7 & 10.2 & 1 \\
8 & 3.3 & 1.80 & 33 & 5.6 & 1.5 & 0.9 & 16.9 & $1-4$ \\
\hline
\end{tabular}

*According to the classification of Hecker (1987).

(see Fig. 2a, where $-\triangle X=-5.5 \mathrm{~cm}$.) The profile measured at $X=-\triangle X$ is referred to hereafter as the intake approach flow. The ADV orientation is accurate within $5^{\circ}$ and the signal to noise ratio was above 8 for the majority of the measurements, which is sufficient for mean velocity measurements.

The channel flow is roughly uniform with moderate left-right asymmetry across the channel crosssection that is probably due to a bend in the feeder pipe. The relative asymmetry is amplified at deeper submergences near the free surface where velocities are very low: this effect may be enhanced by the vortices.

\subsection{PTV Measurements}

An optical method such as PTV is preferred to measure velocities in the vortices because they are small and easily perturbed. The intensity and position of the vortices also fluctuate over time-scales of a few seconds. PTV is accessible and allows the velocity to be measured at many points across the vortex over time-scales ( 0.3 to 2 seconds) that are sufficiently small compared to the vortex time-scale. Appropriate film segments are selected by assessing vortex stability from the shape of the free surface depression, which is visible on the particle images.

Velocities are measured in the right vortex by tracking particles (Pliolite VT) that are injected into the vortex just below the free surface and filmed through the outflow wall with a high-speed camera (Fastec Troubleshooter). The particles are injected at the centre of the vortex to get a more clearly defined profile in the vortex core $\left(r<r_{o}\right)$, since velocities there have the greatest impact on the free surface depression. The vortex is lit from above and below to compensate for light refracted by the free surface depression. The particles have diameters ranging from 150 to $350 \mu \mathrm{m}$ so each particle covers 0.6 to 1.6 pixels in the image. They have a density of $1.003 \mathrm{~kg} \cdot \mathrm{m}^{-3}$ and a terminal velocity of 0.6 to $1.3 \mathrm{~mm} \cdot \mathrm{s}^{-1}$ in still $12^{\circ} \mathrm{C}$ water. The images are recorded at 250 frames per second except for the deepest submergence where 50 frames per second is more appropriate for the much lower velocities. 
The particles are tracked using an open-source particle-tracking code developed by Sbalzarini and Koumoutsakos (2005) that was modified for this experiment to optimize tracking. Figure 3 shows (a) a sample film frame with black and white inverted, (b) the graphical output from the particle-tracking code, and (c) a single plotted trajectory. The transverse coordinate of the particles follows a sinusoidal path in time, of amplitude $2 r_{j}$ and period $\tau_{j}$, which is computed for every complete trajectory cycle $j$ found in the image sequence. Assuming that the azimuthal velocity is steady and axisymmetric and that $V_{\theta} \gg V_{r}, V_{\theta}$ can be computed as $V_{\theta, j}\left(r_{j}\right)=2 \pi r_{j} \tau^{-1}$. Burgers's model (equation 1) can be rewritten in terms of the frequency $\tau$ and fitted to the measured velocities by adjusting $r_{o}$ and $\Gamma_{\infty}$.

(a)

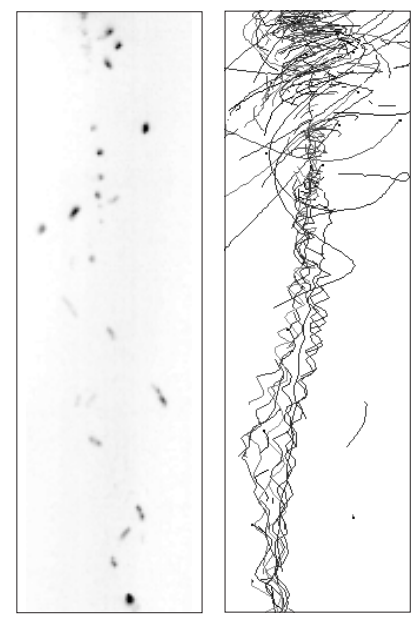

(c)

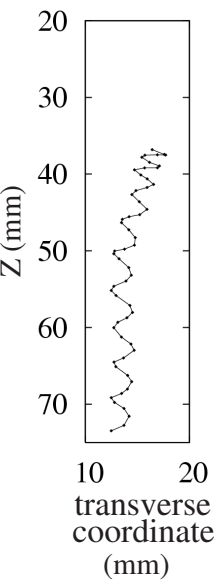

Figure 3 (a) Sample PTV film frame, (b) particle tracking output, (c) sample plotted trajectory.

Fitting Burgers's model to plots of $\left(r_{j}, \tau_{j}\right)$ or the inverse $\left(r_{j}, \tau_{j}^{-1}\right)$ yields different values for $r_{o}$ and $\Gamma_{\infty}$ since points at large radii are weighted more heavily when fitting to $\tau$ and smaller radius points are weighted more heavily for $\tau^{-1}$. The results of both fitting approaches are indicated as error bars to the mean of the two in plots of measured $r_{o}$ and $\Gamma_{\infty}$ values (Figs. 6 to 8). A greater difference between the two values obtained

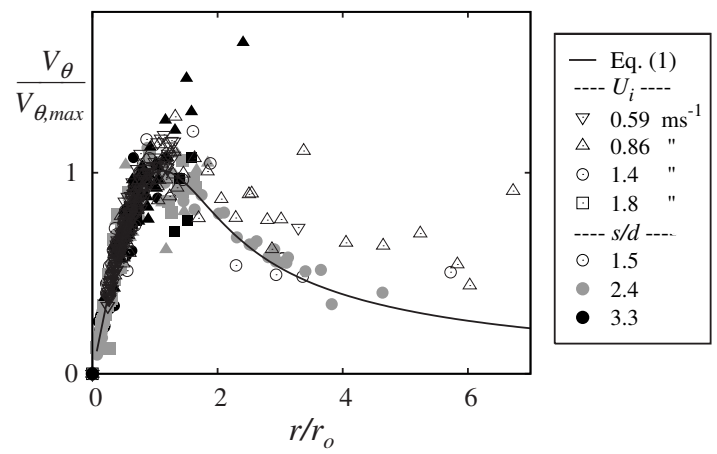

Figure 4 The radial profiles of $V_{\theta}$ measured using PTV for the eight operating conditions. 
suggests greater deviation from Burgers's profile. Figure 4 shows the 18 radial profiles of azimuthal velocity $V_{\theta}\left(r / r_{o}\right) / V_{\theta, \max }$ that were measured for the eight operating conditions, with Burgers's profile (equation 1) plotted as a solid line. The data points measured outside the vortex core $\left(r_{j}>r_{o}\right)$ tend to stray further from the model profile, possibly due to the non-axisymmetric geometry or greater turbulence there.

The axial velocity $V_{z}$ is computed directly from the vertical displacement of particles averaged over three frames. $V_{z}$ fluctuates significantly as the particles complete each circuit around the vortex so the mean slope $\partial V_{z} / \partial z$ is obtained by fitting a straight line through the oscillating signal. Beyond optical effects, deviation of the $V_{z}$ field from axisymmetry may be due to the presence of the wall or pier, curvature of the vortex, or long-wave oscillations travelling along its axis (Crow 1970; Jacquin and Pantano 2002). The slopes of the non-dimensionalized profiles obtained in this manner seem quite consistent for each submergence over different flow rates, despite considerable scatter of the individual velocity points (see Fig. 5b). A few profiles that cover the full vortex length are obtained by tracking $\mathrm{TiO}_{2}$ powder 'dye' injected into the vortex core from the free surface to the intake pipe for three conditions (the highest flow rates at each submergence level). These profiles are consistent with those obtained using PTV and appear in Fig. 5b as well.

\section{Analysis and discussion}

In this section, the vortices observed in the experiment are qualitatively described and a theoretical model is developed by combining Burgers's vortex model (equation 1) with simple analytical relations that link the characteristic radius $r_{o}$, and the bulk circulation $\Gamma_{\infty}$ to the intake approach flow and intake geometry. The analytical relations are adjusted to the particular intake geometry using empirical coefficients fitted to measurements from the experiment. The final model predicts the range of $r_{o}, \Gamma_{\infty}$ and tip depth values that one should expect for a given set of flow conditions for this configuration.

\subsection{Qualitative description}

The vortices produced at the lowest submergence are the strongest and most persistent and those formed at the deepest submergence are the weakest and least persistent. Table 1 indicates the strongest vortex type observed (Hecker 1987). The vortices at the low and medium submergences produce a visible depression of the free surface with a tip depth $h_{0}$ (maximum depression) ranging from a few millimeters to a deep funnel more than a centimeter deep that periodically entrains air bubbles at the highest intake velocity. The vortices produced at the deepest submergence $(s / d=3.3)$ produce such a small depression that they can only be detected from the deformation of reflections on the free surface. At greater flow rates, they rarely last more than a few seconds before dissipating and they require many minutes to form again, but they occasionally entrain floating particles. They also appear to be more strongly influenced by minor asymmetries in the approach flow. At the lowest flow rate for the deepest submergence, the vortices produce a more coherent and long-lasting dye core; this is probably due to lower background turbulence levels and associated perturbations.

\subsection{Axial flow gradients and the vortex characteristic radius}

This section demonstrates how the characteristic radius $r_{o}$ can be predicted from the gradients of the flow outside the vortex. $r_{o}$ is shown to be quite well predicted by the ratio of the molecular viscosity $v$ to the axial gradient of the axial velocity $a=\partial V_{z} / \partial z$ inside the vortex near the free surface. Next, the axial gradient $a$ 
inside the vortex is shown to be driven by the vertical gradient of the intake approach velocity $\partial|U| / \partial Z$ outside the vortex. Finally, the non-dimensional intake approach velocity $|U| / U_{i}$ for all the operating conditions can be written as a simple analytical function of the non-dimensional distance from the inlet $\eta / d$, with two empirical coefficients that account for the specific geometry of the intake. In order to present the resulting vortex model in a more logical sequence, these three elements are presented below in the reverse order: from the structure of the intake approach flow to the flow inside the vortex to the characteristic radius.

First, mean vertical gradients in the intake approach flow are examined. We find that the flow bracketed by the piers can be sufficiently well described for this purpose as a slice of two-dimensional flow into a horizontal line sink located at the upper edge of the intake opening, as shown in Fig. 2a (Bøhling et al. 2010; Yildirim et al. 2000). The upper boundary formed by the free surface causes the flow to deviate from that into a pure line sink. At the deepest submergence, the approach velocity approaches zero at the free surface and begins to recirculate slightly about a horizontal axis formed by the line where the free surface meets the downstream wall. The non-dimensionalized intake approach velocities $|U| / U_{i}$ collapse onto a single line given by

$$
\frac{|U|_{f i t}(\eta)}{U_{i}}=\frac{\mathrm{c}_{1} d}{4 k}\left(\frac{d}{\eta}-\mathrm{c}_{2}\right),
$$

where $|U|=\left(U_{X}^{2}+U_{Y}^{2}+U_{Z}^{2}\right)^{1 / 2}$ and $\eta$ is the total distance from the top of the inlet opening to each measurement point, so that $\eta=\left[(s-Z)^{2}+(\triangle X)^{2}\right]^{1 / 2}$ (see Fig. 2a). The non-dimensional coefficients $\mathrm{c}_{1}=0.8$ and $c_{2}=0.28$ are selected to produce the best fit to the data. Figure 5 a shows the measured velocities as points and the curve fit $|U|_{f i t} / U_{i}$ as a solid line. The point $\eta / d=\mathrm{c}_{2}=0.28$ where the line crosses the graph's vertical axis roughly corresponds to the point where recirculation begins to occur at the free surface for the deepest submergence.

(a)

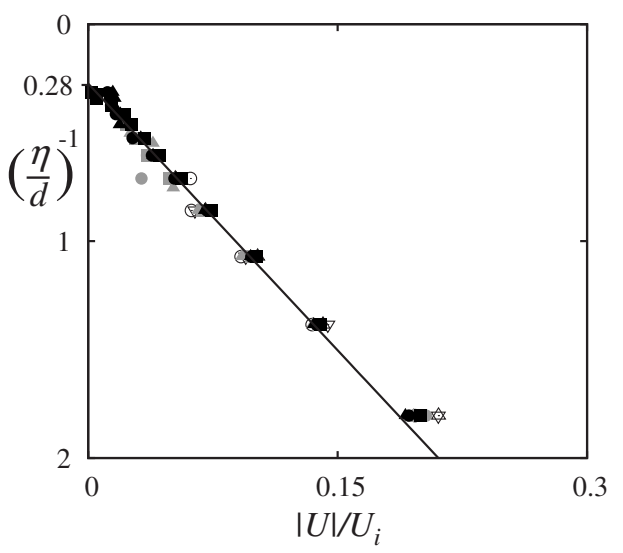

(b)

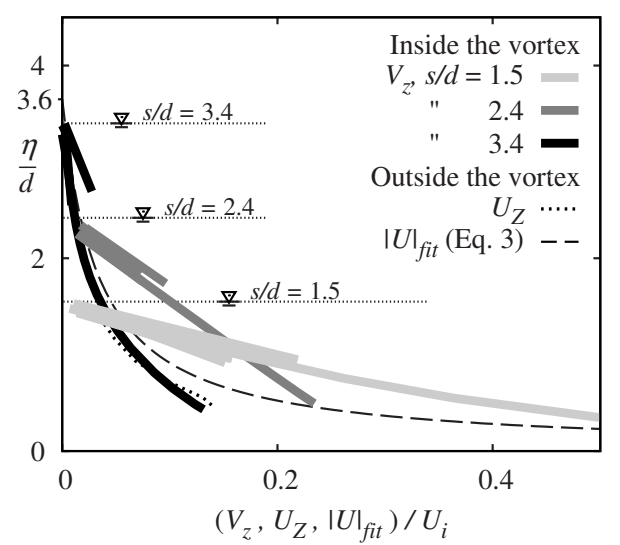

Figure 5 (a) The mean magnitude of velocity $|U| / U_{i}$ outside the vortex, measured by ADV (symbols) and estimated (line) using equation (3). (b) Comparison of the vertical velocity profiles measured inside $\left(V_{Z}\right)$ and outside the vortex $\left(|U|_{f i t}\right.$ and $\left.U_{Z}\right)$. Adapted from (Suerich-Gulick et al. 2014a). 
Next, we examine the link between the intake approach flow (outside the vortex) and the axial flow inside the vortex. Although the vortex is not perfectly vertical along its full length, it is close enough to vertical near the free surface, which is the zone of interest. As shown in Fig. 5(b), the measurements reveal that the mean gradient of the axial flow $V_{z}$ inside the vortex is driven by the mean vertical gradient of the velocity outside the vortex. However $V_{z}$ tends towards a more linear profile for the low and medium submergences, producing much higher axial velocities and gradients near the free surface inside the vortex than outside it. The thick solid lines in Fig. 5(b) show the axial velocity $V_{z} / U_{i}$ measured inside the vortex, while the dashed line shows $|U|_{f i t} / U_{i}$, as defined in equation (3). The location of the free surface is indicated for each submergence; moving upward on the graph to larger values of $\eta / d$ indicates a greater vertical distance from the intake pipe.

The central jet of higher $V_{z}$ inside the vortex following a linear axial profile resembles the axial velocity field produced by Rossi et al.'s (2004) analytical/numerical model and the simulation results of Bøhling et al. (2010). It is possible that the linear axial profile of $V_{z}$ forms inside the vortex because it is more stable than a non-linear one. Pressure may play a role in pushing the axial profile of $V_{z}$ from a non-linear one towards a linear one: the tighter vortex produced by the steeper axial gradient near the intake opening should produce a greater pressure local drop due to centripetal acceleration in the vortex that could drive greater axial velocity from the weaker gradient zone near the free surface to the stronger gradient region closer to the intake pipe. $V_{z}$ appears to stay closer to the velocity $|U|$ outside the vortex for the deepest submergence, developing a linear profile only under some conditions. The effect of the pressure gradient may be too weak under these conditions to push the axial velocity towards a more linear one. Alternatively, the linear axial profile may not have time to form if the vortices have a shorter lifespan, or if the gradient $\partial V_{z} / \partial z$ (Nolan 2001) or the circulation (Jacquin and Pantano 2002) are not strong enough to stabilize the vortices in the presence of the radial gradient in $V_{z}$.

The axial gradient of $V_{z}$ near the free surface is significant because it determines the characteristic radius $r_{o}$ of the vortex in combination with the molecular viscosity $v$. As shown in Figure 6(a), $r_{o}$ extracted from the measured azimuthal velocity profile (Fig. 4) is well predicted by Burgers's model $r_{o}=2(v / a)^{1 / 2}$, with $a=\partial V_{z} / \partial z$ extracted from the same particle trajectories as $r_{o}$. Previous authors proposed using an effective turbulent viscosity $v_{\text {eff }}$ to predict $r_{o}$ (Odgaard 1986; Hite and Mih 1994), but here the molecular $v$ predicts $r_{o}$ quite well even with surrounding turbulent flow, similarly to the laminar case studied by Petitjeans (2003). This supports the hypothesis that radial turbulent mixing is suppressed inside the vortex by the flow rotation, as has been extensively documented in airplane wing tip vortices and other applications (Spalart 1998; Jacquin and Pantano 2002), where the spreading rate of vortices in turbulent flow has been shown to be governed by viscous diffusion, not turbulent diffusion (Cotel and Breidenthal 1999). It would appear that turbulence is suppressed to a significant degree within intake vortices as well, given that dye injected into the vortex produces a clearly delimited dye core (Anwar 1983; Hecker 1987; Schäfer and Hellman 2005), but sufficient turbulence may persist to partially suppress diffusion and hence affect $r_{o}$. This question is discussed further in (Suerich-Gulick et al. 2014b).

Greater scatter and deviation from the predicted value of $r_{o}$ is observed for the deep submergence (black data points), where $r_{o}$ is both more sensitive to variations in $a$ and where the slope of $V_{z}(z)$ is very small and difficult to distinguish from the azimuthal oscillations. It is much more difficult to assess the stability of these shorter-lived vortices since they don't deform the free surface enough to be visible from 
the side. These vortices may therefore not have reached the equilibrium state assumed in Burgers's model, and $a$ may vary considerably with time for a given operating condition as the vortex forms, strengthens and weakens.

(a)

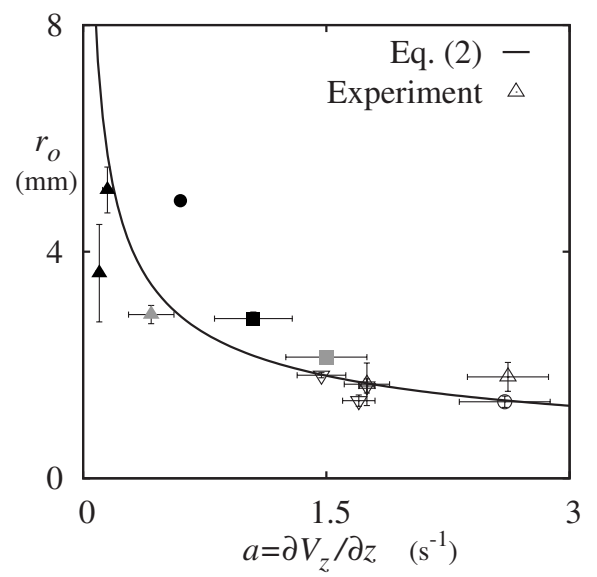

(b)

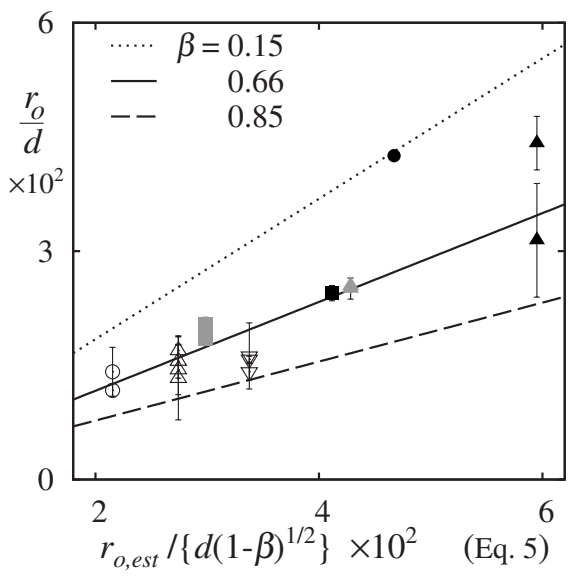

Figure 6 (a) Dependence of the characteristic radius $r_{o}$ on the axial velocity gradient $a=\partial V_{z} / \partial z$ near the free surface inside the vortex. $r_{o}$ measured by PTV (symbols) is compared to the estimate (line) given by equation (2) using the measured gradient $a$. (b) Dependence of $r_{o}$ on the geometry and flow conditions. Measured values (symbols) are compared to estimates (lines) obtained from equation (5). See Fig. 4 for the key to operating conditions.

We have established that $r_{o}$ is controlled by $v$ and $\partial V_{z} / \partial z$ and that the profile $V_{z}(z)$ is driven by the intake approach velocity. $r_{o}$ can therefore now be predicted as a function of the submergence $s / d$ and the intake velocity $U_{i}$ (Fig. 6b). Given the uncertainty as to how much $V_{z}(z)$ will tend towards a more linear profile, a range of values for $r_{o}$ is predicted instead of a fixed value. We assume that the axial velocity $V_{z}$ inside the vortex matches the magnitude of velocity $|U|$ outside the vortex at the free surface and at a distance $z=\beta s$ below the free surface, following a linear profile between the two points. The non-dimensional coefficient $\beta$, ranging from 0 to 0.85 , indicates how far the linear profile extends below the free surface. $\beta=0.66$ indicates that the linear profile extends $2 / 3$ of the distance from the free surface to the top of the intake pipe and $\beta=0$ indicates that $V_{z}$ matches the $|U|$ profile along the full length of the vortex. $|U|_{f i t}$ is estimated using equation (3) with $\eta \approx s$ at the free surface and $\eta \approx(1-\beta) s$ at $z=\beta s$, producing

$$
a_{e s t}=\frac{c_{1} U_{i} d^{2}}{4 k s^{2}(1-\beta)} .
$$

The resulting characteristic radius $r_{o, \text { est }}$ is obtained by substituting $a_{\text {est }}$ into equation (2):

$$
r_{o, e s t}=\frac{4 s}{d}\left(\frac{v k(1-\beta)}{\mathrm{c}_{1} U_{i}}\right)^{1 / 2} .
$$

When $V_{z}(z)$ follows the outside profile $|U|(Z)$ over most of the vortex length (corresponding to a smaller value of $\beta$ ), then the axial gradient $a=\partial V_{z} / \partial z$ at the free surface is weaker, producing a larger characteristic radius $r_{o}$ for a given $U_{i}$ and $s / d$. If a linear profile forms over a greater proportion of the flow 
(larger $\beta$ ), then a greater $a$ at the free surface will produce a smaller characteristic radius. Figure $6 \mathrm{~b}$ compares the values of $r_{o} / d$ measured in the experiment to the envelope of values estimated from equation (5) using different values of $\beta(0.15,0.66,0.85)$. The solid line $\beta=0.66$ roughly corresponds to the profiles observed in Fig. $5 \mathrm{~b}$ for $s / d=2.4$ and some cases of $s / d=1.5 . \beta=0.15$ (dotted line) and $\beta=0.85$ (dashed line) roughly bracket the measured values that lie above and below this mean, respectively.

In this graph and the next, all the measurements for a given operating condition are vertically aligned, so the significant variability of $r_{o}$ within one operating condition is clearly visible. Once again the measured values of $r_{o}$ for the deep submergence (black points), stray furthest from the mean value in Fig. 6b. It is likely that some of this variability is due to variations in the axial velocity profile inside the vortex.

\subsection{Bulk circulation $\Gamma_{\infty}$}

The bulk circulation $\Gamma_{\infty}$ is controlled by the interaction of the channel flow with the intake geometry, as vorticity generated upstream or near the intake becomes concentrated into a vortex above the intake pipe through axial stretching (Quick 1970; Suerich-Gulick et al. 2014a). $\Gamma_{\infty}$ is estimated by integrating $V_{\theta}$ along the full circumference of a circle of radius $r \gg r_{o}$ (Thomson 1869) since the bulk of vorticity is concentrated in $r<r_{o}: \Gamma_{\infty}=\Gamma\left(r \gg r_{o}\right)=\int_{0}^{2 \pi} V_{\theta} r \mathrm{~d} \theta$. The two free surface vortices are roughly confined to the wake of the pier here in all but one case, so the integral is computed along a circle of diameter $l_{p}$ inscribed inside the region delimited by the pier and the downstream wall (see Fig. 2b). The azimuthal velocity on this circle should be proportional to the mean horizontal free surface velocity measured directly in front of the intake, which is roughly equal to $|U|_{\text {fit }}$ at $\eta=s$, since $U_{Z}=0$ at the free surface. $\Gamma_{\infty}$ is therefore estimated as $\pi l_{p} U_{f i t, \eta=s}$. As was the case for $r_{o}$, there are significant variations in $\Gamma_{\infty}$ at each operating condition, so we estimate a range of values for $\Gamma_{\infty}$ instead of a fixed value:

$$
\Gamma_{\infty, e s t}=\frac{\mathrm{c}_{3} \mathrm{c}_{1} d U_{i} \pi l_{p}}{4 k}\left(\frac{d}{\mathrm{c}_{4} s}-\mathrm{c}_{2}\right),
$$

where the coefficients $c_{3}$ and $c_{4}$ are fitted to the measured data. $c_{3}=0.33$ and $c_{4}$ takes two values, 1.0 and 0.6 which give the lower $\left(\Gamma_{\infty, \min }\right)$ and upper $\left(\Gamma_{\infty, \max }\right)$ estimated values of $\Gamma_{\infty}$, respectively. The resulting range of values is indicated by the grey boxes in Fig. 7a, while the points show the measured values.

Setting $c_{4}$ to 0.6 instead of 1.0 is roughly equivalent to estimating the circulation from the approach velocity $|U|$ a third of the way down from the free surface instead of directly at the free surface. This suggests that the stronger approach velocity below the free surface may be strengthening the vortex to a greater $\Gamma_{\infty}$ value under certain conditions, for example if the vortex lasts longer. The magnitude of the variations in $\Gamma_{\infty}$ also appears to scale with the channel Reynolds number $R_{c h}$, as shown in Fig. $7 \mathrm{~b}$, so that

$$
\Gamma_{\infty, \max }-\Gamma_{\infty, \text { min }} \approx \mathrm{c}_{5} v \mathrm{R}_{\mathrm{ch}},
$$

where $c_{5}=0.06$ is fitted to the measured data. Perhaps the greater turbulence levels at greater Reynolds numbers produce greater occasional deviations from the mean free surface velocity, generating greater circulation.

In all cases except for case $6\left(U_{i}=0.86, s / d=3.3\right)$, the mean intensity of the two free surface vortices appears to be roughly equal when averaged over time. In case 6 , the mean approach velocity at the 
(a)

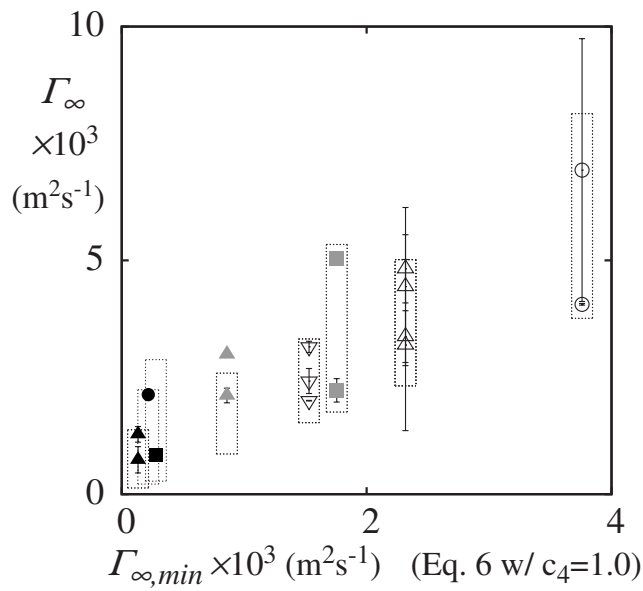

(b)

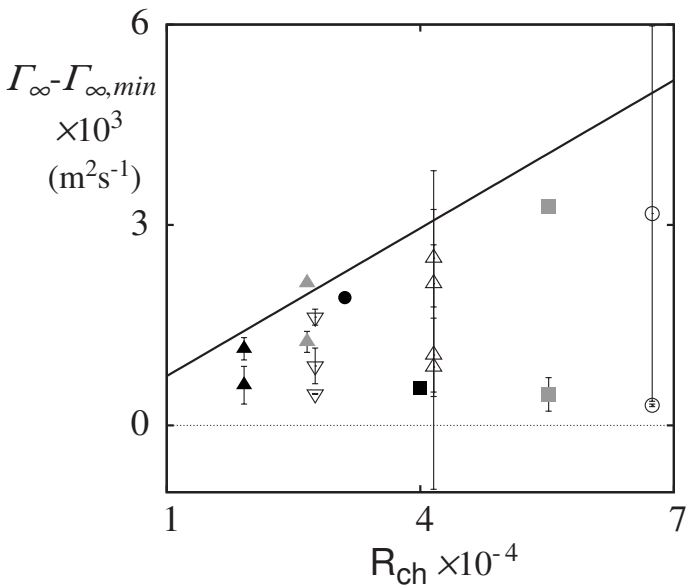

Figure 7 The bulk circulation $\Gamma_{\infty}$. (a) Comparison of measured (symbols) to estimated (boxes) values obtained from equation (6). (b) Variation in measured $\Gamma_{\infty}$ (symbols) as a function of $\mathrm{R}_{\mathrm{ch}}$, with the upper limit (line) estimated using equation (7). See Fig. 4 for the key to operating conditions.

free surface was so weak that the flow structure was qualitatively different from the other cases and only one vortex formed at any given time, halfway between the two piers. The data points for this case are not included in Fig. 7.

\subsection{Free surface depression}

The centripetal acceleration $V_{\theta}^{2} / r$ in the vortex generates a pressure drop in its centre and hence a depression of the free surface whose magnitude is determined by the equilibrium of the forces exerted by gravity, centripetal acceleration, and surface tension. Neglecting surface tension, which is left to a subsequent paper (Suerich-Gulick et al. 2014b), an analytical relation for the tip depth $h_{0}$ is obtained by substituting Burgers's profile (equation 1) for $V_{\theta}(r)$ :

$$
h_{0}=\int_{\infty}^{0}\left(\frac{V_{\theta}(\dot{r})^{2}}{g \dot{r}}\right) \mathrm{d} \dot{r}=\frac{0.17 \Gamma_{\infty}^{2}}{\pi^{2} r_{o}^{2} g},
$$

where $h_{0}$ is the distance from the nominal (undeformed) free surface down to the lowest point of the free surface depression caused by the vortex, and $g$ is the gravitational acceleration.

The tip depth $h_{0, \text { comp }}$ that corresponds to the $V_{\theta}$ profiles measured in the experiment is computed by substituting the measured values of $\Gamma_{\infty}$ and $r_{o}$ into equation (8). It is compared in Fig. 8a to the actual measured tip depth $h_{0, \exp }$ recorded in the same film segments as the particle trajectories used to measure the velocities, allowing us to evaluate if the velocities were properly measured and if Burgers's model is appropriate. The solid line in Fig. 8a indicates exact agreement between $h_{0, \text { comp }}$ and $h_{0, \text { exp }}$; the horizontal error bars show the spread in $r_{o}$ and $\Gamma_{\infty}$ values obtained from the two fitting methods (section 2.3), while the symbols indicate their mean. The $h_{0, \text { comp }}$ values follow the same trend as $h_{0, \exp }$, but the former are larger because they are computed without surface tension, which significantly reduces the total depression in the experiment. 
Alternatively the expressions for $\Gamma_{\infty}$ (equation 6) and $r_{o}$ (equation 5) developed above can be substituted into Burgers's relation (equation 1) for $V_{\theta}(r)$, producing an estimated tip depth $h_{0, \text { est }}$ in terms of the intake conditions and geometry:

$$
\frac{h_{0, e s t}}{d}=\frac{0.17 \Gamma_{\infty}^{2}}{\pi^{2} d r_{o}^{2} g}=\frac{\mathrm{c}_{6} \mathrm{c}_{3}^{2} \mathrm{c}_{1}^{3}}{(1-\beta)} \mathrm{R}_{\mathrm{s}} \mathrm{F}_{\mathrm{s}}^{2}\left(\frac{d}{k}\right)^{3}\left(\frac{l_{p}}{s}\right)^{2}\left(\frac{d}{\mathrm{c}_{4} s}-\mathrm{c}_{2}\right)^{2},
$$

where $\mathrm{c}_{6}=6.6 \times 10^{-4}$ is determined by the integration and $\mathrm{R}_{\mathrm{s}} \mathrm{F}_{\mathrm{s}}^{2}=U_{i}^{3} /(v g)$.

The estimated range of $h_{0, \text { est }} / d$ compares fairly well to the measured values $h_{0, \text { exp }} / d$, as shown in Fig. 8b. The symbols indicate the measured values and the gray boxes indicate the values estimated using equation (9) with $\left(c_{4}=1.0, \beta=0.15\right)$ for the minima, $\left(c_{4}=0.66, \beta=0.75\right)$ for the mid-range values, and $\left(\mathrm{c}_{4}=0.6, \beta=0.85\right)$ for the maxima. There is much greater scatter in this graph, due to the variations in $r_{o}$ and $\Gamma_{\infty}$ discussed above. It is difficult to compare the results presented here to empirical relationships, because most of these relationships predict the critical submergence for air entrainment, whereas the experiment described here focuses mainly on weaker vortices.

(a)

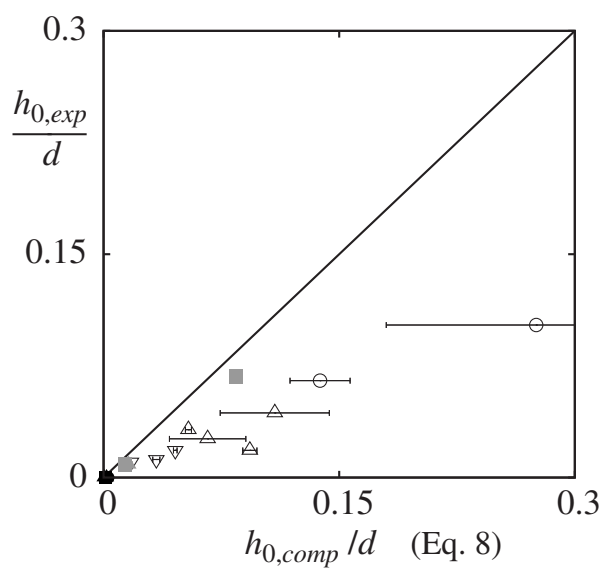

(b)

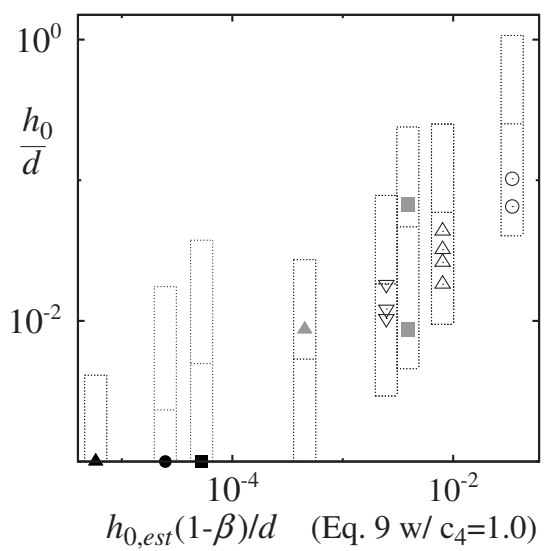

Figure 8 Free surface depression. (a) Correspondence between the measured tip depth $h_{0, \exp }$ and the computed tip depth $h_{0, \text { comp }}$ obtained from equation (8) with the measured $r_{o}$ and $\Gamma_{\infty}$ values. (b) The measured tip depth (symbols) and the predicted limiting values (boxes) estimated from the geometry and flow conditions (equation 9). See Fig. 4 for the key to operating conditions.

\section{Conclusion}

The results presented here establish a clear link between the velocity profile directly in front of the intake and the axial velocity profile $V_{z}(z)$ inside the vortex, highlighting the role of the intake approach flow in driving axial vortex stretching. The measurements strongly suggest that radial turbulent diffusion is effectively suppressed in the vortex core, in agreement with observations in wing tip vortices. It is difficult to predict how much $V_{z}(z)$ will stray from the non-linear profile of the flow outside the vortex towards a more linear one and equally difficult to explain the significant variations in $\Gamma_{\infty}$ observed for a given operating condition. Due to observed variations in the axial profile $V_{z}(z)$ and $\Gamma_{\infty}$, expected ranges for $r_{o}, \Gamma_{\infty}$ and $h_{0}$ are estimated 
as a function of $U_{i}$ and $s / d$ instead of fixed values. These analytical relations are used in a subsequent paper (Suerich-Gulick et al. 2014b) to propose and discuss quantitative relations for translating vortex characteristics observed in a laboratory-scale model to the prototype scale based on the scaling behaviour of surface tension, viscosity and turbulence.

\section{Acknowledgements}

We would like to acknowledge the contributions and help of David Morissette, Pierre Tadeo, Tristan Aubel, Maryse Page, Anne-Marie Giroux and Sébastien Houde.

\section{Funding}

This work was supported by the National Sciences and Engineering Research Council of Canada (Postgraduate Scholarship B), the Fonds Québécois pour les Sciences et les Technologies (Bourse de recherche au doctorat), and Hydro-Québec's research centre.

\section{Notation}

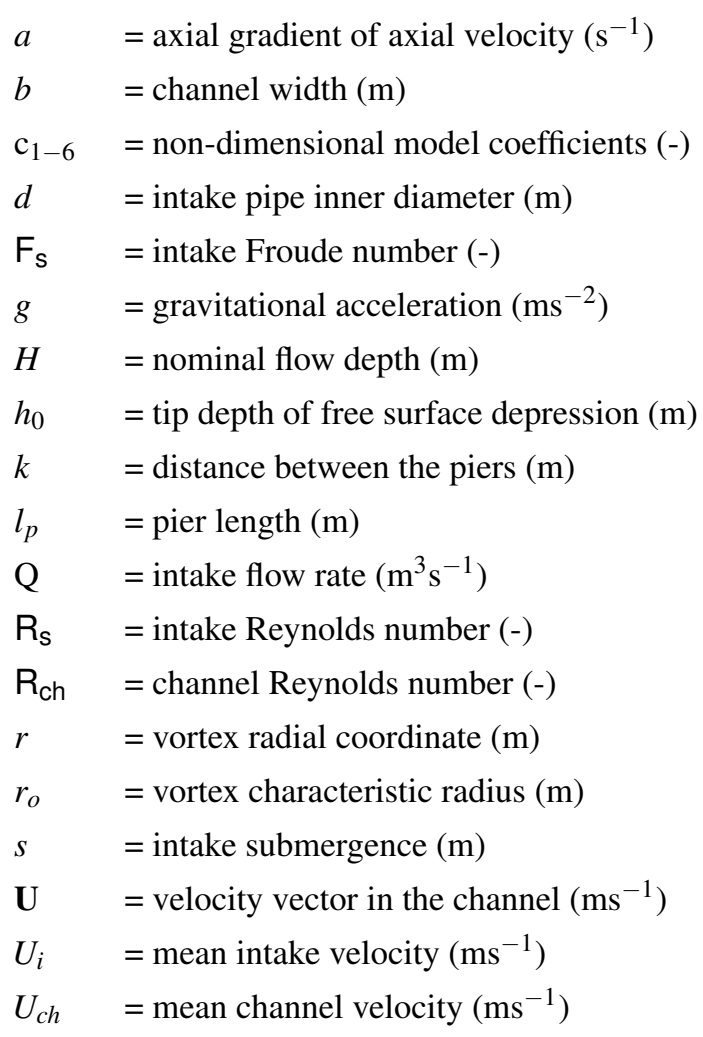




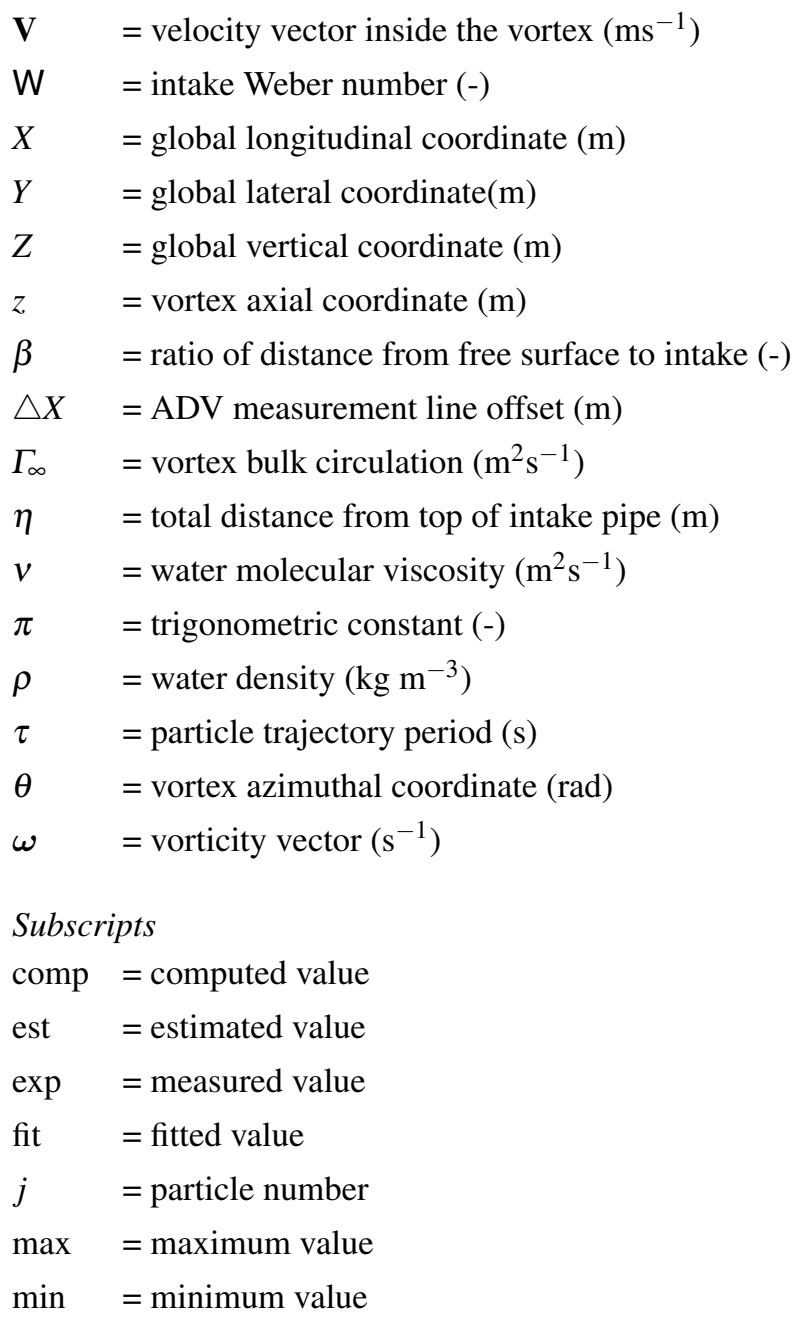

\section{References}

Andersen, A., Bohr, T., Stenum, B., Rasmussen, J.J., Lautrup, B. (2006). The bathtub vortex in a rotating container. J. Fluid Mech. 556, 121-146.

Ansar, M., Nakato, T. (2001). Experimental study of 3D pump-intake flows with and without cross flow. $J$. Hydraulic Eng. 127(10), 825-834.

Anwar, H.O. (1983). The non-dimensional parameters of free-surface vortices measured for horizontal and vertically inverted intakes. Houille Blanche 1, 11-25.

Bøhling, L., Andersen, A., Fabre, D. (2010). Structure of a steady drain-hole vortex in a viscous fluid. J. Fluid Mech. 656, 177-188.

Burgers, J.M. (1948). A mathematical model illustrating the theory of turbulence. Adv. in Appl. Mech. 1, 171-199.

Carriveau, R., Kopp, G.A., Baddour, R.E. (2009). Free-surface stretching-sustained intake vortices. J. Hydraulic Res. 47(4), 486-491.

Cotel, A.J., Breidenthal, R.E. (1999). Turbulence inside a vortex. Phys. Fluids 11(10), 3026-3029. 
Crow, S.C. (1970). Stability theory for a pair of trailing vortices. AIAA J. 8, 2172-2179.

Daggett, L.L., Keulegan, G.H. (1974). Similitude in free-surface vortex formations. J. Hydraul. Div. ASCE 100(HY11), 1565-1581.

Einstein, H.A., Li, H. (1951). Steady vortex flow in a real fluid. In Proc. Heat Trans. and Fluid Mech. Inst., 33-43, Stanford University, Palo Alto, Calif.

Gordon, J.L. (1970). Designing intakes to avoid free-surface vortices. Water Power Apr, 137-138.

Gulliver, J.S., Rindels, A.J., Lindblom, K.C. (1986). Designing intakes to avoid free-surface vortices. Int. Water Power Dam Constr. 38(9), 24-28.

Hecker, G.E. (1987). Fundamentals of vortex intake flow. In J. Knauss, ed., Swirling flow problems at intakes, 13-38, A.A. Balkema, Rotterdam.

Helmholtz, H. (1867). On integrals of the hydrodynamical equations, which express vortex motion. Phil. Mag. S. 4 33(226), 485-512.

Hite, J.E., Mih, W.C. (1994). Velocity of air-core vortices at hydraulic intakes. J. Hydraulic Eng. 120(3), 284-297.

Ito, K., Sakai, T., Eguchi, Y., Monji, H., Ohshima, H., Uchibori, A., Xu, Y. (2010). Improvement of gas entrainment prediction method - introduction of surface tension effect. J. Nucl. Sci. Technol. 47(9), $771-778$

Jacquin, L., Pantano, C. (2002). On the persistence of trailing vortices. J. Fluid Mech. 471, 159-168.

Jain, A.K., Raju, K.G.R., Garde, R.J. (1978). Vortex formation at vertical pipe intakes. J. Hydraul. Div. ASCE 104(HY10), 1429-1445.

Jiming, M., Yuanbo, L., Jitang, H. (2000). Minimum submergence before double-entrance pressure intakes. J. Hydraulic Eng. 126(8), 628-631.

Lundgren, T.S. (1985). The vortical flow above the drain-hole in a rotating vessel. J. Fluid Mech. 155, 381412.

Miles, J. (1998). A note on the Burgers-Rott vortex with a free surface. Z. Angew. Math. Phys. 49, 162-165.

Montilla, G., Marcano, A., Castro, C. (2004). Air entrainment at Guri Dam intake operating at low heads. In Proc. Int. Conf. Hydraulics of Dams and River Structures, Tehran, Iran, 53-60, F. Yazdandoost, J. Attari, eds., Balkema, Rotterdam, NL.

Nakayama, A., Hisasue, N. (2010). Large eddy simulation of vortex flow in intake channel of hydropower facility. J. Hydraulic Res. 48(4), 415-427.

Nolan, D.S. (2001). The stabilizing effects of axial stretching on turbulent vortex dynamics. Phys. Fluids 13(6), 1724-1738.

Odgaard, A.J. (1986). Free-surface air core vortex. J. Hydraulic Eng. 112(7), 610-620.

Pennino, B.J., Hecker, G.E. (1979). A synthesis of model data for pumped storage intakes. In Pump turbine schemes, Joint ASME-CSME Appl. Mech., Fluids Eng. and Bioeng. Conf., 103-112, Niagara Falls, NY.

Petitjeans, P. (2003). Stretching of a vortical structure: filaments of vorticity. Europhysics News 34(1), 20-23.

Quick, M.C. (1970). Efficiency of air-entraining vortex formation at water intake. J. Hydraulic Eng. 96(HY7), 
1403-1416.

Rossi, M., Bottausci, F., Maurel, A., Petitjeans, P. (2004). A nonuniformly stretched vortex. Phys. Rev. Lett. 92(5), 054504/1-4.

Rott, N. (1958). On the viscous core of a line vortex. Z. Angew. Math. Phys. 9b, 543-553.

Sbalzarini, I.F., Koumoutsakos, P. (2005). Feature point tracking and trajectory analysis for video imaging in cell biology. J. Struct. Biology 151, 182-195.

Schäfer, F., Hellman, D.H. (2005). Optimization of approach flow conditions of vertical pumping systems by physical model investigation. In Proc of ASME FEDSM2005, Houston, USA.

Spalart, P.R. (1998). Airplane trailing vortices. Ann. Rev. Fluid Mech. 30, 107-138.

Stepanyants, Y., Yeoh, G. (2008). Stationary bathtub vortices and a critical regime of liquid discharge. J. Fluid Mech. 604, 77-98.

Suerich-Gulick, F., Gaskin, S.J., Parkinson, E., Villeneuve, M. (2014a). The characteristics of free surface vortices at low-head hydropower intakes. J. Hydraulic Eng. (in press) .

Suerich-Gulick, F., Gaskin, S.J., Villeneuve, M., Parkinson, E. (2014b). Free surface intake vortices: Scale effects due to surface tension and viscosity. Accepted for publication in the J. Hydraulic Res. .

Tastan, K., Yildirim, N. (2010). Effects of dimensionless parameters on air-entraining vortices. J. Hydraulic Res. 48(1), 57-64.

Thomson, W.T. (1869). On vortex motion. Trans. Roy. Soc. Edinb. 25, 217-260.

Wang, Y., Jiang, C., Liang, D. (2011). Comparison between empirical formulae of intake vortices. J. Hydraulic Res. 49(1), 113-116.

Yildirim, N., Kocabaş, F., Gülcan, S. (2000). Flow-boundary effects on critical submergence of intake pipe. J. Hydraulic Eng. 126(4), 288-297. 